\title{
Heptametallic Bowl-Shaped Complexes Derived From Conjugated Schiff-base Macrocycles: Synthesis, Characterization, and X-ray Crystal Structures
}

Amanda J. Gallant, Jonathan H. Chong, and Mark J. MacLachlan*

Department of Chemistry, University of British Columbia, 2036 Main Mall, Vancouver, BC, V6T 1Z1 CANADA.

Supporting Information

\section{Table of Contents}

Experimental Section $\quad \mathrm{S} 2$

NMR Simulation $\quad$ S3

X-Ray Diffraction Studies $\quad$ S4

References and Footnotes $\quad$ S5

Figure S1. ${ }^{1} \mathrm{H}$ NMR spectrum 3a $\quad$ S6

Figure S2. ${ }^{13} \mathrm{C}$ NMR spectrum 3a $\quad$ S6

Figure S3. MALDI-TOF spectrum of 3a $\quad$ S7

Figure S4. ESI mass spectrum of 3a $\quad$ S8

Figure S5. ${ }^{1} \mathrm{H}$ NMR spectrum $\mathbf{3 b} \quad$ S9

Figure S6. ${ }^{13} \mathrm{C}$ NMR spectrum $3 \mathbf{b} \quad$ S9

Figure S7. ${ }^{13} \mathrm{C}$ APT NMR spectrum $\mathbf{3 b} \quad \mathrm{S} 10$

Figure S8. ESI mass spectrum of $\mathbf{3 b} \quad \mathrm{S} 10$

Figure S9. ${ }^{1} \mathrm{H}$ NMR spectrum 3c $\quad$ S11

Figure S10. ${ }^{13} \mathrm{C}$ NMR spectrum 3c $\quad \mathrm{S} 11$

Figure S11. ${ }^{13} \mathrm{C}$ APT NMR spectrum 3c $\quad \mathrm{S} 12$

Figure S12. ESI mass spectrum of 3c $\quad \mathrm{S} 12$ 


\section{Experimental Section}

Materials. Zinc(II) acetate dihydrate and $\left(\mathrm{CDCl}_{2}\right)_{2}$ were obtained from Aldrich. Ethanol was distilled from magnesium under $\mathrm{N}_{2}$. Macrocycles 1a-c were prepared according to our literature method. ${ }^{1}$

Equipment. All reactions were carried out under air unless otherwise noted. ${ }^{1} \mathrm{H}$ and ${ }^{13} \mathrm{C}$ NMR spectra were recorded on either a Bruker AV-300 or AV-400 spectrometer. ${ }^{13} \mathrm{C}$ NMR spectra were recorded using either a proton decoupled or APT pulse sequence. ${ }^{1} \mathrm{H}$ and ${ }^{13} \mathrm{C}$ NMR spectra were calibrated to the residual protonated solvent at $\delta 7.24$ and $\delta$ $77.00 \mathrm{ppm}$, respectively, in $\mathrm{CDCl}_{3}$. UV-vis spectra were obtained in $\mathrm{CH}_{2} \mathrm{Cl}_{2}$ (ca. $5 \times 10^{-6}$ M) on a Varian Cary 5000 UV-vis-near-IR spectrophotometer using a $1 \mathrm{~cm}$ quartz cuvette. IR spectra were obtained as $\mathrm{KBr}$ discs with a Bomems MB-series spectrometer. MALDI-TOF mass spectra were obtained in a dithranol matrix (cast from THF) at the UBC Microanalytical Services Laboratory on a Bruker Biflex IV instrument. Electrospray ionization (ESI) mass spectra were obtained on a Bruker Esquire LC instrument. The samples were analyzed in $\mathrm{MeOH}: \mathrm{CHCl}_{3}(1: 1)$ at $100 \mu \mathrm{M}$. Elemental analyses $(\mathrm{C}, \mathrm{H}, \mathrm{N}, \mathrm{Zn})$ were performed at Canadian Microanalytical Services (Delta, BC) and the UBC Microanalytical Services Laboratory. Melting points were obtained on a Fisher-John's melting point apparatus.

We have learned that in general, mass spectrometry of these complexes rarely gives the molecular ion. Most of the major peaks observed by MALDI or ESI techniques can be assigned to sensible fragments. In the ESI of the complexes, $\left[\mathrm{M}-\mathrm{Zn}{ }_{2} \mathrm{O}(\mathrm{OAc})_{2}+\mathrm{Na}\right]^{+}$is usually the major fragment observed.

Synthesis of Heptazinc Complex $3 \mathbf{b}\left(\mathbf{R}={ }^{\mathbf{n}} \mathbf{C}_{5} \mathbf{H}_{11}\right)$. Zinc acetate dihydrate (374 $\mathrm{mg}$, $1.70 \mathrm{mmol})$ was added to a solution of $\mathbf{1 b}(250 \mathrm{mg}, 0.202 \mathrm{mmol})$ in $50 \mathrm{~mL}$ of dry ethanol under $\mathrm{N}_{2}$. The deep red solution turned bright orange within a few minutes at room temperature. After stirring at reflux $\left(\sim 90^{\circ} \mathrm{C}\right)$ for $2 \mathrm{~h}$, the orange mixture was cooled to room temperature and the precipitate isolated by filtration. The crude solid was recrystallized from ethanol to afford an orange microcrystalline solid $\mathbf{3 b}$. Yield: $307 \mathrm{mg}$ (0.15 mmol, 74\%).

Data for $3 \mathbf{b}\left(\mathbf{R}={ }^{\mathbf{n}} \mathbf{C}_{5} \mathbf{H}_{11}\right) \cdot{ }^{13} \mathrm{C} \mathrm{NMR}\left(100.7 \mathrm{MHz}, \mathrm{CDCl}_{3}\right) \delta 180.0\left(\mathrm{O}_{2} \mathrm{CCH}_{3}\right), 178.8$ $\left(\mathrm{O}_{2} \mathrm{CCH}_{3}\right), 161.9,158.6,150.2,134.1,120.8,119.3,102.0,69.7\left(\mathrm{OCH}_{2}\right), 28.9\left(\mathrm{CH}_{2}\right)$, $28.2\left(\mathrm{CH}_{2}\right), 23.4\left(\mathrm{O}_{2} \mathrm{CCH}_{3}\right), 22.4\left(\mathrm{CH}_{2}\right), 21.7\left(\mathrm{O}_{2} \mathrm{CCH}_{3}\right), 14.0\left(\mathrm{CH}_{2} \mathrm{CH}_{3}\right) .{ }^{1} \mathrm{H}$ NMR (300 $\left.\mathrm{MHz} \mathrm{CDCl}_{3}\right) \delta 8.33(\mathrm{~s}, 6 \mathrm{H}$, imine), 6.99 (s, 6H, Ar), $6.72(\mathrm{~s}, 6 \mathrm{H}, \mathrm{Ar}), 4.08(\mathrm{~m}, 12 \mathrm{H}$, $\left.\mathrm{OCH}_{2}\right), 1.95\left(\mathrm{~s}, 9 \mathrm{H}, \mathrm{O}_{2} \mathrm{CCH}_{3}\right), 1.89\left(\mathrm{~m}, 12 \mathrm{H}, \mathrm{CH}_{2}\right), 1.86\left(\mathrm{~s}, 9 \mathrm{H}, \mathrm{O}_{2} \mathrm{CCH}_{3}\right), 1.62\left(\mathrm{~s}, \mathrm{H}_{2} \mathrm{O}\right)$, $1.47\left(\mathrm{~m}, 24 \mathrm{H}, \mathrm{CH}_{2}\right), 0.97\left(\mathrm{t},{ }^{3} \mathrm{~J}_{\mathrm{HH}}=7.1 \mathrm{~Hz}, 18 \mathrm{H}, \mathrm{CH}_{2} \mathrm{CH}_{3}\right)$. UV-vis $\left(\mathrm{CH}_{2} \mathrm{Cl}_{2}\right) \lambda_{\max }(\varepsilon)=$ $415\left(1.2 \times 10^{5}\right), 347.5\left(6.4 \times 10^{4}\right), 240\left(7.4 \times 10^{4}\right) \mathrm{nm}\left(\mathrm{L} \mathrm{mol}^{-1} \mathrm{~cm}^{-1}\right)$. ESI-MS: $\mathrm{m} / \mathrm{z}=1810$ $\left.\left([3 \mathbf{b}]-\mathrm{Zn}_{2} \mathrm{O}(\mathrm{OAc})_{2}+\mathrm{Na}^{+}\right), 1870\left([3 \mathbf{b}]-\mathrm{Zn}(\mathrm{OAc})_{2}+\mathrm{H}^{+}\right), 1994([3 \mathbf{b}]-\mathrm{OAc})^{-}\right) . \quad \mathrm{IR}(\mathrm{KBr}): \mathrm{v}=$ 3451, 2960, 2932, 2860, 1612, 1572, 1503, 1455, 1448, 1399, 1318, 1262, 1215, 1185 , $1104,1036,927,751,690.2 \mathrm{~cm}^{-1}$. Mp. $>280{ }^{\circ} \mathrm{C}$. Anal. Calc'd for $3 \mathbf{b}: \mathrm{C}_{84} \mathrm{H}_{102} \mathrm{~N}_{6} \mathrm{O}_{25} \mathrm{Zn}_{7}$ : C, 49.13; H, 5.01; N, 4.09. Found: C, 49.23; H, 5.08; N, 4.40. Single crystals suitable for $\mathrm{X}$-ray diffraction were obtained from benzene. 
Synthesis of Heptazinc Complex 3a $\left(\mathbf{R}=\mathbf{C}_{2} \mathbf{H}_{5}\right)$. Heptazinc complex 3a was prepared by a procedure analogous to that for $\mathbf{3 b}$. The product was recrystallized from DMF. Yield was ca. $80 \%$.

Data for 3a $\left(\mathbf{R}=\mathbf{C}_{2} \mathbf{H}_{5}\right) \cdot{ }^{13} \mathrm{C}$ NMR $\left(75.5 \mathrm{MHz}, \mathrm{DMF}-\mathrm{d}_{7}\right) \delta 178.8\left(\mathrm{O}_{2} \mathrm{CCH}_{3}\right), 173.5$ $\left(\mathrm{O}_{2} \mathrm{CCH}_{3}\right), 163.7,158.4,150.3,134.7,121.7,120.5,102.9,65.2\left(\mathrm{OCH}_{2}\right), 15.0\left(\mathrm{CH}_{2} \mathrm{CH}_{3}\right)$. ${ }^{1} \mathrm{H}$ NMR (300 MHz, DMF-d $) \delta 8.80$ (s, 6H, imine), 7.53 (s, 6H, Ar), 6.90 (s, 6H, Ar), $4.30\left(\mathrm{~m}, 12 \mathrm{H}, \mathrm{OCH}_{2}\right), 1.88\left(\mathrm{~s}, 9 \mathrm{H}, \mathrm{O}_{2} \mathrm{CCH}_{3}\right), 1.71\left(\mathrm{~s}, 9 \mathrm{H}, \mathrm{O}_{2} \mathrm{CCH}_{3}\right), 1.46(\mathrm{t}, 18 \mathrm{H}$, $\left.\mathrm{CH}_{2} \mathrm{CH}_{3}\right)$. UV-vis $\left(\mathrm{CH}_{2} \mathrm{Cl}_{2}\right) \lambda_{\max }(\varepsilon)=413\left(1.2 \times 10^{5}\right), 347\left(6.6 \times 10^{4}\right), 242\left(7.4 \times 10^{4}\right)$ $\mathrm{nm}\left(\mathrm{L} \mathrm{mol}^{-1} \mathrm{~cm}^{-1}\right)$. ESI-MS: $\mathrm{m} / \mathrm{z}=1557.2\left([3 \mathrm{a}]-\mathrm{Zn}_{2} \mathrm{O}(\mathrm{OAc})_{2}+\mathrm{Na}^{+}\right)$. MALDI-TOF: $\mathrm{m} / \mathrm{z}=$ $1557 \quad\left([3 \mathbf{3 a}]-\mathrm{Zn}_{2} \mathrm{O}(\mathrm{OAc})_{2}+\mathrm{Na}^{+}\right), \quad 1741 \quad\left([3 \mathbf{3 a}]-\mathrm{OAc}^{-}\right), \quad 1820 \quad\left([3 \mathbf{3}]+\mathrm{H}_{2} \mathrm{O}+\mathrm{H}^{+}\right), \quad 1899$ $\left([3 \mathrm{a}]+\left(\mathrm{H}_{2} \mathrm{O}\right)_{3}+\left(\mathrm{Na}^{+}\right)_{2}-\mathrm{H}^{+}\right) . \quad$ IR $(\mathrm{KBr}): v=2979,2923,2894,1609,1562,1505,1458$, 1444, 1392, 1316, 1264, 1217, 1179, 1108, 1037, 938, $754 \mathrm{~cm}^{-1}$. Mp. $\sim 250{ }^{\circ} \mathrm{C}$ (dec.) Anal. Calc'd for $3 \mathrm{a}^{\cdot} 2 \mathrm{H}_{2} \mathrm{O}: \mathrm{C}_{66} \mathrm{H}_{70} \mathrm{~N}_{6} \mathrm{O}_{25} \mathrm{Zn}_{7}$ : C, 43.15; H, 3.84; N, 4.57. Found: $\mathrm{C}, 42.93$; H, 4.05; N, 5.02 (water observed in NMR spectrum). Single crystals suitable for X-ray diffraction were obtained from DMSO.

Synthesis of the Heptazinc Complex $3 c\left(R={ }^{n} \mathbf{C}_{6} \mathbf{H}_{13}\right)$. Heptazinc complex $3 c$ was prepared by a procedure analogous to that for $\mathbf{3 b}$. The product was recrystallized from ethanol. Yield was ca. $45 \%$.

Data for 3c ( $\left.\mathbf{R}={ }^{\mathbf{n}} \mathbf{C}_{\mathbf{6}} \mathbf{H}_{13}\right) \cdot{ }^{13} \mathrm{C}$ NMR $\left(300 \mathrm{MHz}, \mathrm{CDCl}_{3}\right) \delta 180.1\left(\mathrm{O}_{2} \mathrm{CCH}_{3}\right), 178.8$ $\left(\mathrm{O}_{2} \mathrm{CCH}_{3}\right), 161.9,158.7,150.3,134.2,120.8,119.3,102.0,69.7\left(\mathrm{OCH}_{2}\right), 31.5\left(\mathrm{CH}_{2}\right)$, $29.2\left(\mathrm{CH}_{2}\right), 25.7\left(\mathrm{CH}_{2}\right), 23.4\left(\mathrm{O}_{2} \mathrm{CCH}_{3}\right), 22.6\left(\mathrm{CH}_{2}\right), 21.6\left(\mathrm{O}_{2} \mathrm{CCH}_{3}\right), 14.0\left(\mathrm{CH}_{2} \mathrm{CH}_{3}\right) .{ }^{1} \mathrm{H}$ NMR $\left(300 \mathrm{MHz}, \mathrm{CDCl}_{3}\right) \delta 8.31(\mathrm{~s}, 6 \mathrm{H}$, imine), $6.97(\mathrm{~s}, 6 \mathrm{H}$, aromatic $\mathrm{CH}), 6.69(\mathrm{~s}, 6 \mathrm{H}$, aromatic $\mathrm{CH}), 4.07\left(\mathrm{~m}, 12 \mathrm{H}, \mathrm{OCH}_{2}\right), 1.93\left(\mathrm{~s}, 9 \mathrm{H}, \mathrm{O}_{2} \mathrm{CCH}_{3}\right), 1.86\left(\mathrm{~m}, 12 \mathrm{H}, \mathrm{CH}_{2}\right), 1.84(\mathrm{~s}$, $\left.9 \mathrm{H}, \mathrm{O}_{2} \mathrm{CCH}_{3}\right), 1.53\left(\mathrm{~m}, 24 \mathrm{H}, \mathrm{CH}_{2}\right), 1.36\left(\mathrm{~m}, 12 \mathrm{H}, \mathrm{CH}_{2}\right), 0.91\left(\mathrm{t},{ }^{3} \mathrm{~J}_{\mathrm{HH}}=6.7 \mathrm{~Hz}, \mathrm{CH}_{2} \mathrm{CH}_{3}\right)$. UV-vis $\left(\mathrm{CH}_{2} \mathrm{Cl}_{2}\right) \lambda_{\max }(\varepsilon)=415\left(1.3 \times 10^{5}\right), 346(6.9 \times 104), 241(7.8 \times 104) \mathrm{nm}\left(\mathrm{L} \mathrm{mol}^{-1}\right.$ $\left.\mathrm{cm}^{-1}\right)$. ESI-MS: $m / z=1893.8\left([3 \mathrm{c}]-\mathrm{Zn}_{2} \mathrm{O}(\mathrm{OAc})_{2}+\mathrm{Na}\right)$. IR $(\mathrm{KBr}): v=2953,2929,2860$, $1617,1563,1507,1462,1447,1396,1319,1268,1222,1183,1166,1111,1017,722 \mathrm{~cm}^{-}$ 1. Mp. $\sim 250{ }^{\circ} \mathrm{C}$ (dec.). Anal. Calc'd for 3c: $\mathrm{C}_{90} \mathrm{H}_{114} \mathrm{~N}_{6} \mathrm{O}_{25} \mathrm{Zn}_{7}$ : C, 50.57; H, 5.38; N, 3.93; Zn, 21.4. Found: C, 50.41; H, 5.41; N, 3.89; Zn, 20.30.

NMR Simulation. ${ }^{1} \mathrm{H}$ NMR data of the $\mathrm{OCH}_{2}$ group in $3 \mathrm{c}$ were simulated on a $\mathrm{PC}$ as an $\mathrm{ABX}_{2}$ spin system with MestRe-C v2.3a (C. Cobas, J. Cruces, and F.J. Sardina, Universidad de Santiago de Compostela, Spain).

${ }^{1} \mathrm{H}$ NMR data were simulated as an $\mathrm{ABX}_{2}$ spin system using $\delta 4.044\left(\mathrm{H}_{\mathrm{A}}\right), 4.102\left(\mathrm{H}_{\mathrm{B}}\right)$, $1.80\left(\mathrm{H}_{\mathrm{X}}\right) ;{ }^{2} \mathrm{~J}_{\mathrm{AB}}=8.70 \mathrm{~Hz} ;{ }^{3} \mathrm{~J}_{\mathrm{AX}}=6.60 \mathrm{~Hz} ;{ }^{3} \mathrm{~J}_{\mathrm{BX}}=6.40 \mathrm{~Hz}$. Peaks Calc'd (Found): 1242.45 (1242.71), 1236.06 (1236.29), 1233.81 (1233.60), 1227.35 (1227.25), 1223.23 (1223.18), 1220.97 (1220.97), 1216.65 (1216.66), 1210.02 (1210.03), 1207.94 (1207.72), $1201.27(1200.78) \mathrm{Hz}$. 
X-Ray Diffraction Study of 3a. Crystals of 3a suitable for X-ray diffraction were grown from DMSO by ether diffusion. All measurements were made on a Rigaku/ADSC diffractometer with graphite monochromated $\mathrm{Mo}-\mathrm{K}_{\alpha}$ radiation $(\lambda=0.71073 \AA)$. The data was collected at a temperature of $-100.0 \pm 0.1^{\circ} \mathrm{C}$ to a maximum $2 \theta$ value of $52.04^{\circ}$. Data were collected in a series of $\phi$ and $\omega$ scans in $0.50^{\circ}$ oscillations with $59 \mathrm{~s}$ exposures. Of the 60844 reflections that were collected, 27033 were unique $\left(R_{i n t}=0.0756\right)$; equivalent reflections were merged. The data was collected using $\mathrm{d}^{*} \mathrm{TREK}^{4}$ and integrated using the CrystalClear software package. ${ }^{5}$ Data were corrected for absorption effects using a multi-scan technique (CrystalClear), with max and min transmission coefficients of 1.0000 and 0.7855 , respectively. The data was corrected for Lorentz and polarization effects. The structure was solved by direct methods. ${ }^{6}$ The SQUEEZE ${ }^{7}$ function in PLATON $^{8}$ was used to remove approximately 3 disordered DMSO molecules per asymmetric unit. All non-hydrogen atoms were refined anisotropically. All hydrogen atoms were included in calculated positions but were not refined. The final cycle of fullmatrix least-squares refinement on $\mathrm{F}^{2}$ was based on 27033 reflections and 1202 variable parameters and converged (largest parameter shift was 0.001 times its esd). ${ }^{9}$

Crystal data: red prism $(0.50 \times 0.30 \times 0.20 \mathrm{~mm}), \mathrm{a}=12.0360(11) \AA, \mathrm{b}=26.181(3)$ $\AA, c=26.547(3) \AA, \alpha=118.824(3)^{\circ}, \beta=93.440(3)^{\circ}, \gamma=90.154(3)^{\circ}, V=7310.9(14) \AA^{3}$, space group P-1 (triclinic), $\mathrm{Z}=4$, mol. wt. $=2239.87, \mathrm{~T}=-100.0(1){ }^{\circ} \mathrm{C}, \mu\left(\mathrm{Mo}-\mathrm{K}_{\alpha}\right)=$ $12.67 \mathrm{~cm}^{-1}, 60844$ observed (27033 unique) reflections, 1202 variables, $\mathrm{R}_{1}=0.1035$, $\mathrm{wR}_{2}$ $=0.2056, \mathrm{GOF}=0.962$.

X-Ray Diffraction Study of $\mathbf{3 b}$. Crystals of $\mathbf{3 b}$ suitable for X-ray diffraction were grown from benzene. All measurements were made on a Bruker X8 diffractometer with graphite monochromated Mo- $\mathrm{K}_{\alpha}$ radiation $(\lambda=0.71073 \AA)$. The data was collected at a temperature of $-100.0 \pm 0.1^{\circ} \mathrm{C}$ to a maximum $2 \theta$ value of $47.60^{\circ}$. Data were collected in a series of $\phi$ and $\omega$ scans in $0.50^{\circ}$ oscillations with 20s exposures. Of the 90372 reflections that were collected, 15013 were unique $\left(\mathrm{R}_{\mathrm{int}}=0.0749\right)$; equivalent reflections were merged. Data was collected and integrated using the Bruker SAINT ${ }^{10}$ software package. The data was corrected for absorption effects using the multi-scan technique (SADABS), ${ }^{11}$ with $\max$ and min transmission coefficients of 0.916 and 0.720 , respectively. The data were corrected for Lorentz and polarization effects. The structure was solved by direct methods. ${ }^{6}$ The SQUEEZE ${ }^{7}$ function in PLATON ${ }^{8}$ was used to remove approximately 2 disordered benzene molecules per asymmetric unit. The carbons on some of the alkoxy groups were disordered and modeled in two orientations. All nonhydrogen atoms were refined anisotropically. All hydrogen atoms were included in calculated positions but not refined. The final cycle of full-matrix least-squares refinement on $\mathrm{F}^{2}$ was based on 15013 reflections and 1031 variable parameters and converged (largest parameter shift was 4.243 times its esd (one of the free variables for refining the disorder alkyl chains - all other parameters were near $0 \times$ ESD)). ${ }^{9}$ Further details are available in the supplementary CIF file.

Crystal data: red needle $(0.30 \times 0.10 \times 0.05 \mathrm{~mm}), \mathrm{a}=27.342(5) \AA, \mathrm{b}=38.204(5) \AA$, $\mathrm{c}=20.526(5), \beta=113.343(5)^{\circ}, \mathrm{V}=19686(7) \AA^{3}$, space group $\mathrm{C} 2 / \mathrm{c}$ (monoclinic), $\mathrm{Z}=4$, mol. wt. $=2053.46, \mathrm{~T}=-100.0(1){ }^{\circ} \mathrm{C}, \mu\left(\mathrm{Mo}_{\mathrm{\alpha}}\right)=17.46 \mathrm{~cm}^{-1}, 90372$ observed $(15013$ unique) reflections, 1031 variables, $\mathrm{R}_{1}=0.1013$, $\mathrm{wR}_{2}=0.1489, \mathrm{GOF}=1.015$. 


\section{References and Footnotes}

[1] Gallant, A. J.; Hui, J. K. H.; Zahariev, F.; Wang, Y. A.; MacLachlan, M. J. J. Org. Chem. 2005, 70, 7936-7946.

[2] Stewart, J. J. P. J. Comp. Chem. 1989, 10, 209.

[3] Gaussian 03, Revision B.05, M. J. Frisch et al., Gaussian, Inc., Pittsburgh PA, 2003.

[4] d*TREK. Area Detector Software. Version 7.1I. Molecular Structure Corporation (2001).

[5] CrystalClear 1.3.5 SP2. Molecular Structure Corporation (2003).

[6] SIR92. Altomare, A.; Cascarano, M.; Giacovazzo, C.; Guagliardi, A. J. Appl. Cryst. 2004, 26, 343.

[7] SQUEEZE - Sluis, P. v. d.; Spek,A. L. Acta Crystallogr., Sect A 1990, 46, 194-201. [8] PLATON, A Multipurpose Crystallographic Tool, Utrecht University, Utrecht, The Netherlands, A. L. Spek, (1998).

[9] Least squares function minimized:

$$
\Sigma w\left(\mathrm{~F}_{\mathrm{o}}{ }^{2}-\mathrm{F}_{\mathrm{c}}{ }^{2}\right)^{2} .
$$

[10] SAINT. Version 6.02. Bruker AXS Inc., Madison, Wisconsin, USA. (1999).

[11] SADABS. Bruker Nonius area detector scaling and absorption correction - V2.05, Bruker AXS Inc., Madison, Wisconsin, USA. 


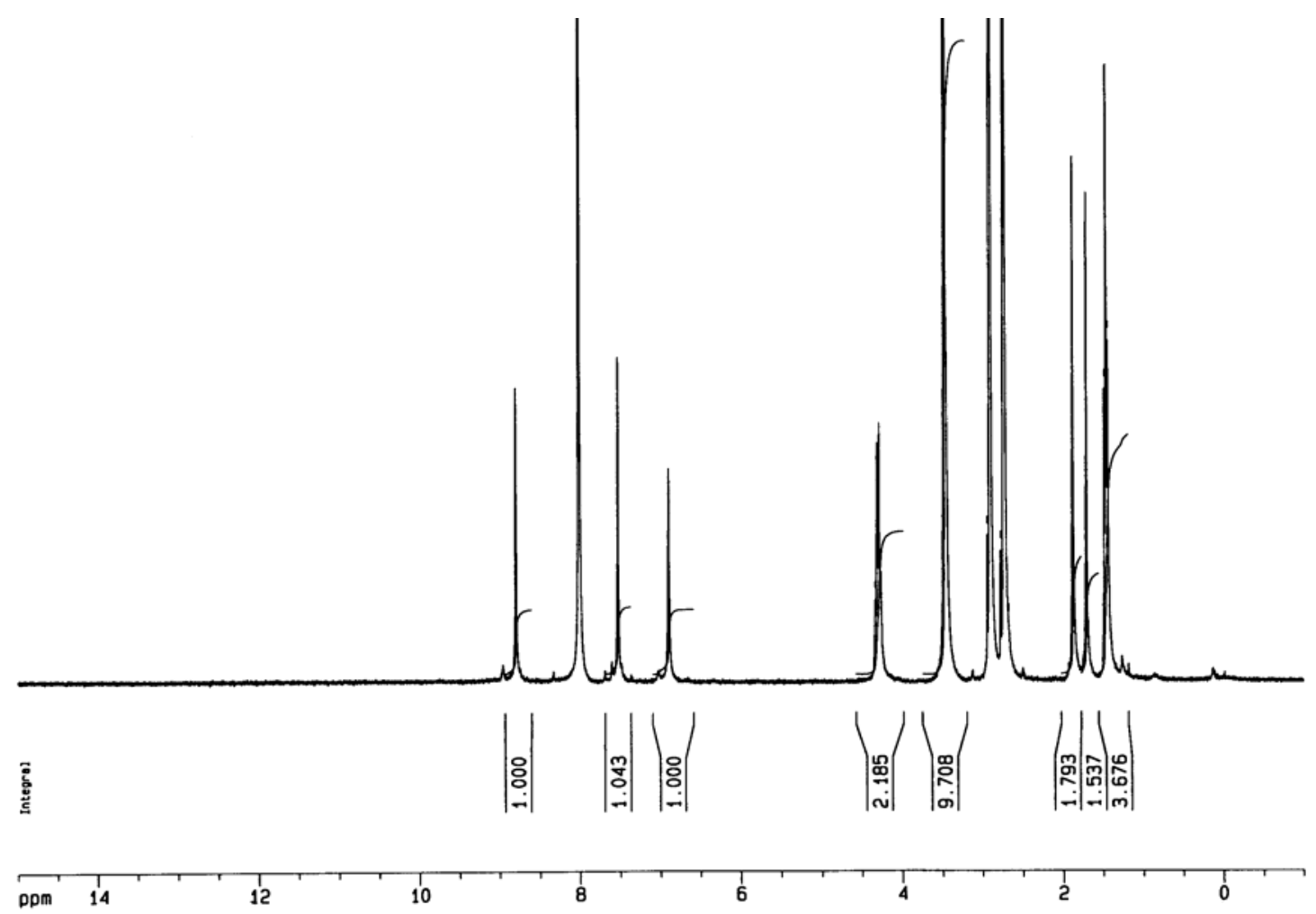

Figure S1. ${ }^{1} \mathrm{H}$ NMR spectrum $\left(300 \mathrm{MHz}, \mathrm{DMF}-\mathrm{d}_{7}\right)$ of $\mathbf{3 a}\left(\mathrm{R}=\mathrm{OC}_{2} \mathrm{H}_{5}\right)$.

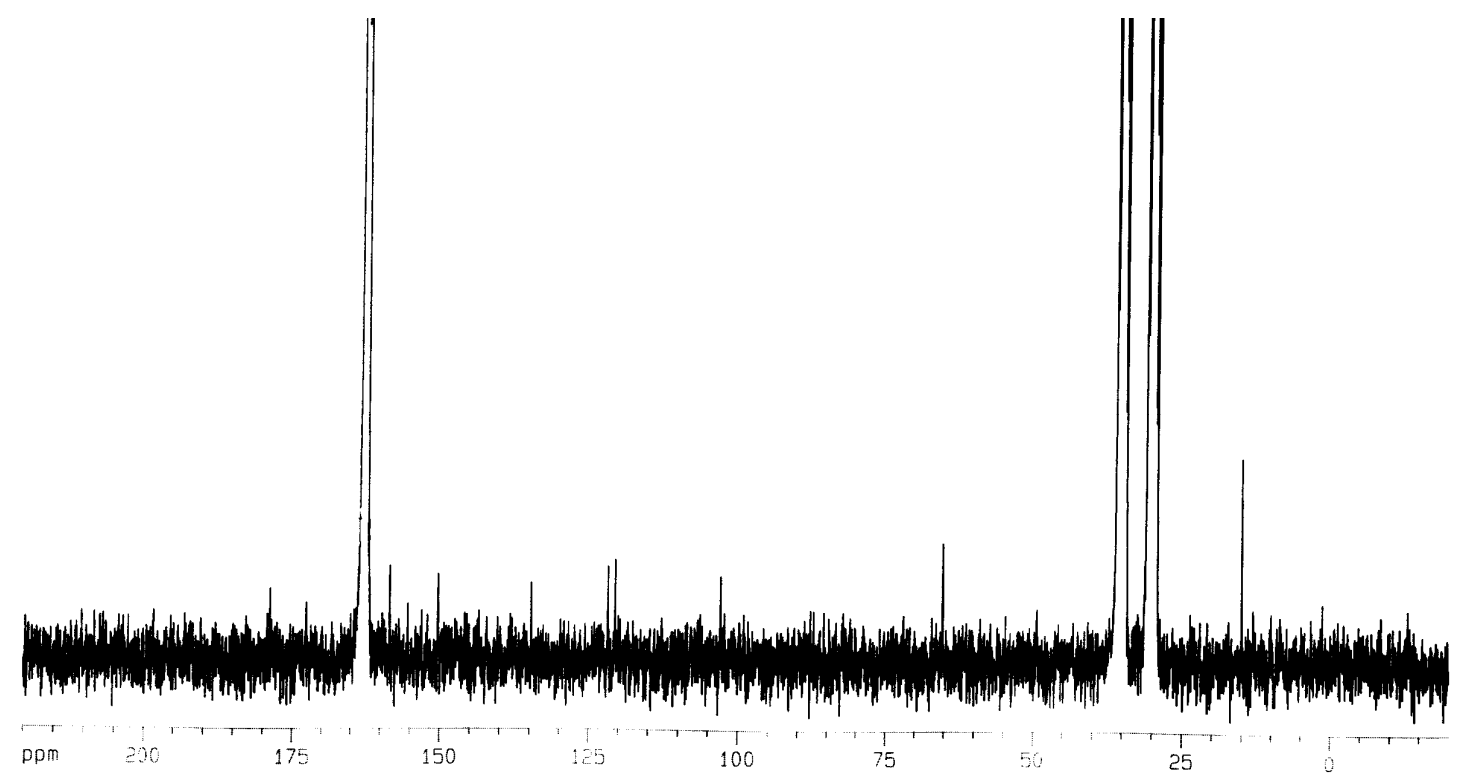

Figure S2. ${ }^{13} \mathrm{C}$ NMR spectrum $\left(75.5 \mathrm{MHz}, \mathrm{DMF}-\mathrm{d}_{7}\right)$ of $\mathbf{3 a}\left(\mathrm{R}=\mathrm{OC}_{2} \mathrm{H}_{5}\right)$. 


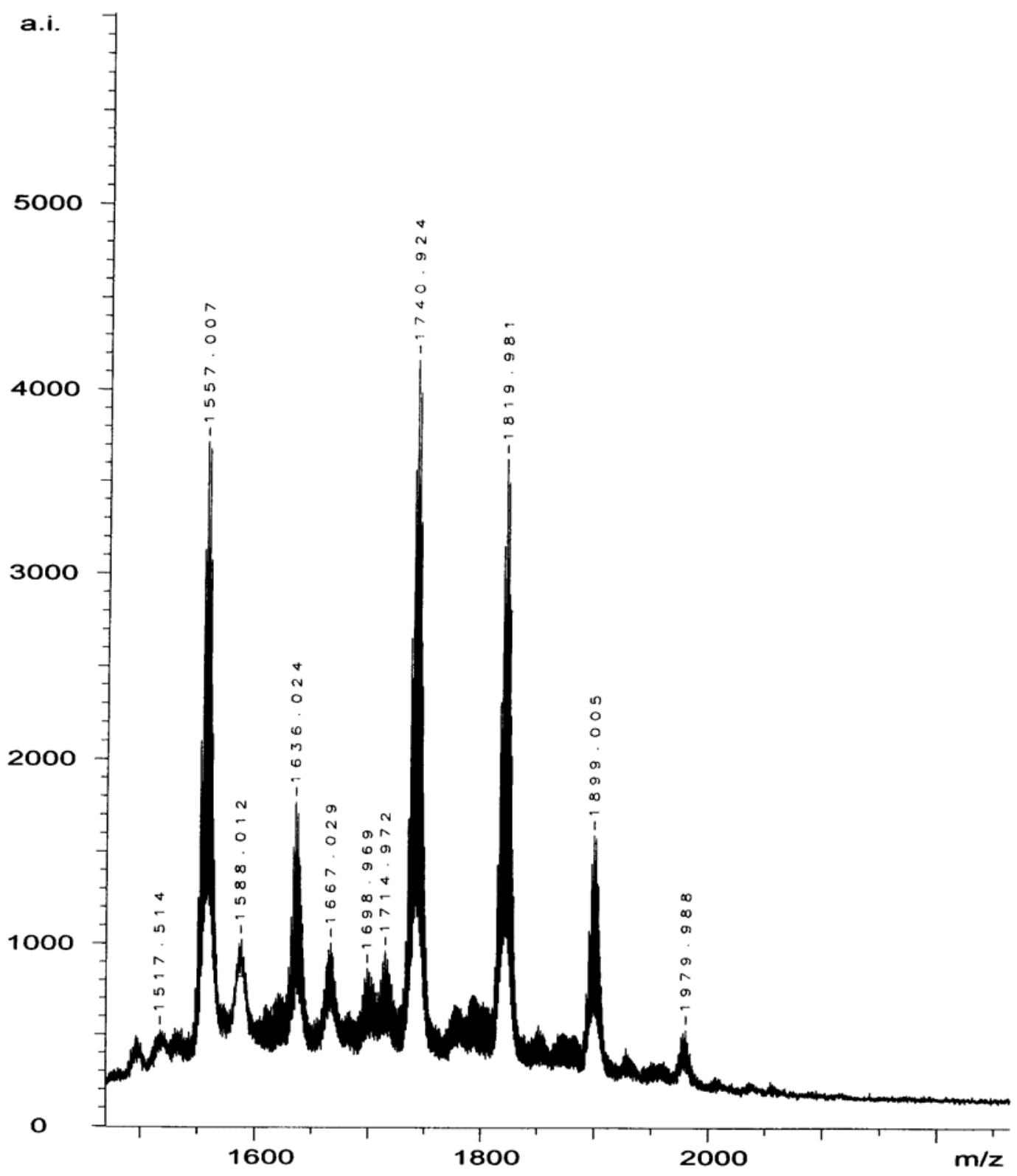

Figure S3. MALDI-TOF spectrum of $3 \mathbf{a}\left(\mathrm{R}=\mathrm{OC}_{2} \mathrm{H}_{5}\right)$. 


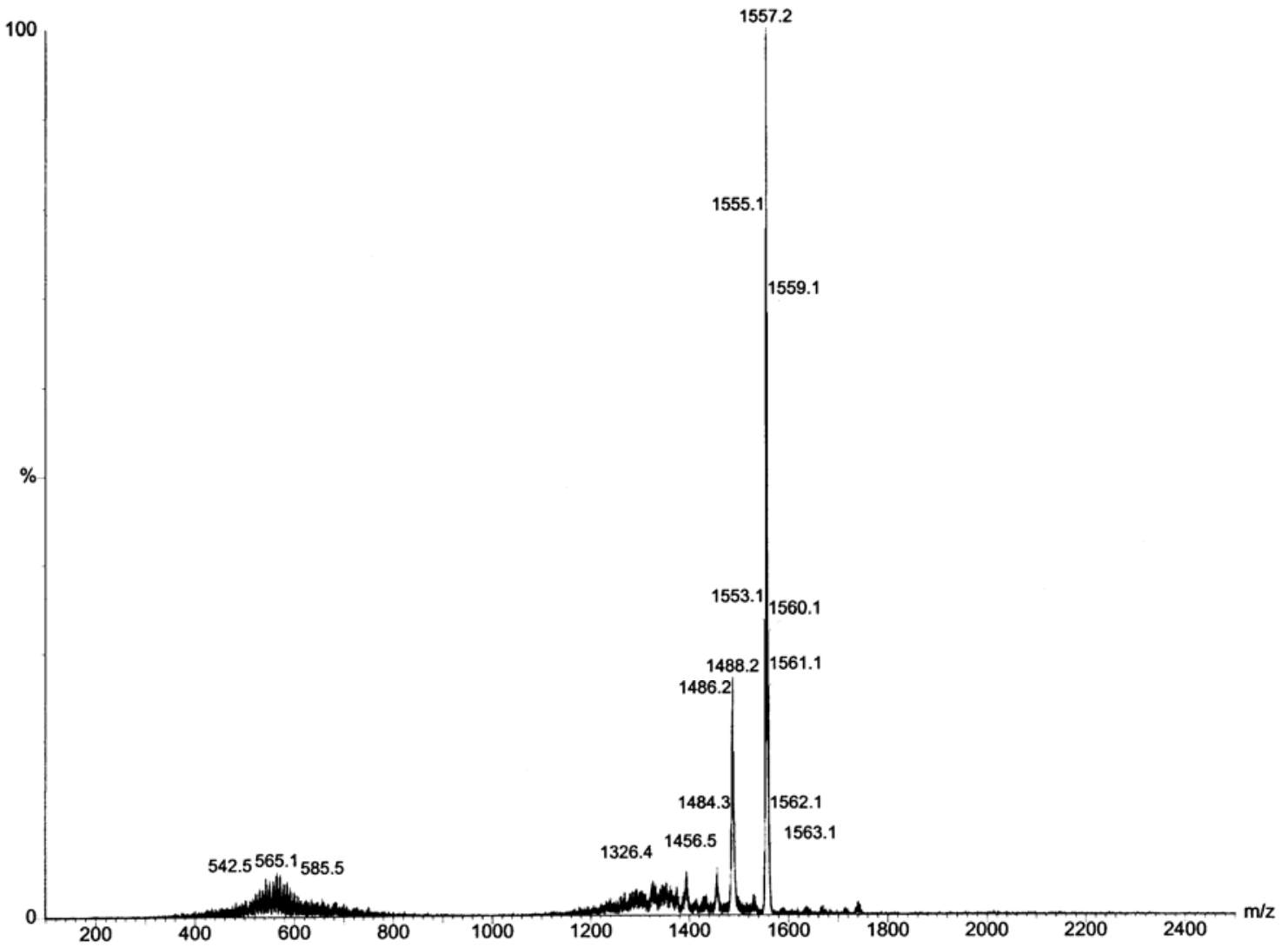

Figure S4. ESI mass spectrum of $\mathbf{3 a}\left(\mathrm{R}=\mathrm{OC}_{2} \mathrm{H}_{5}\right)$. 


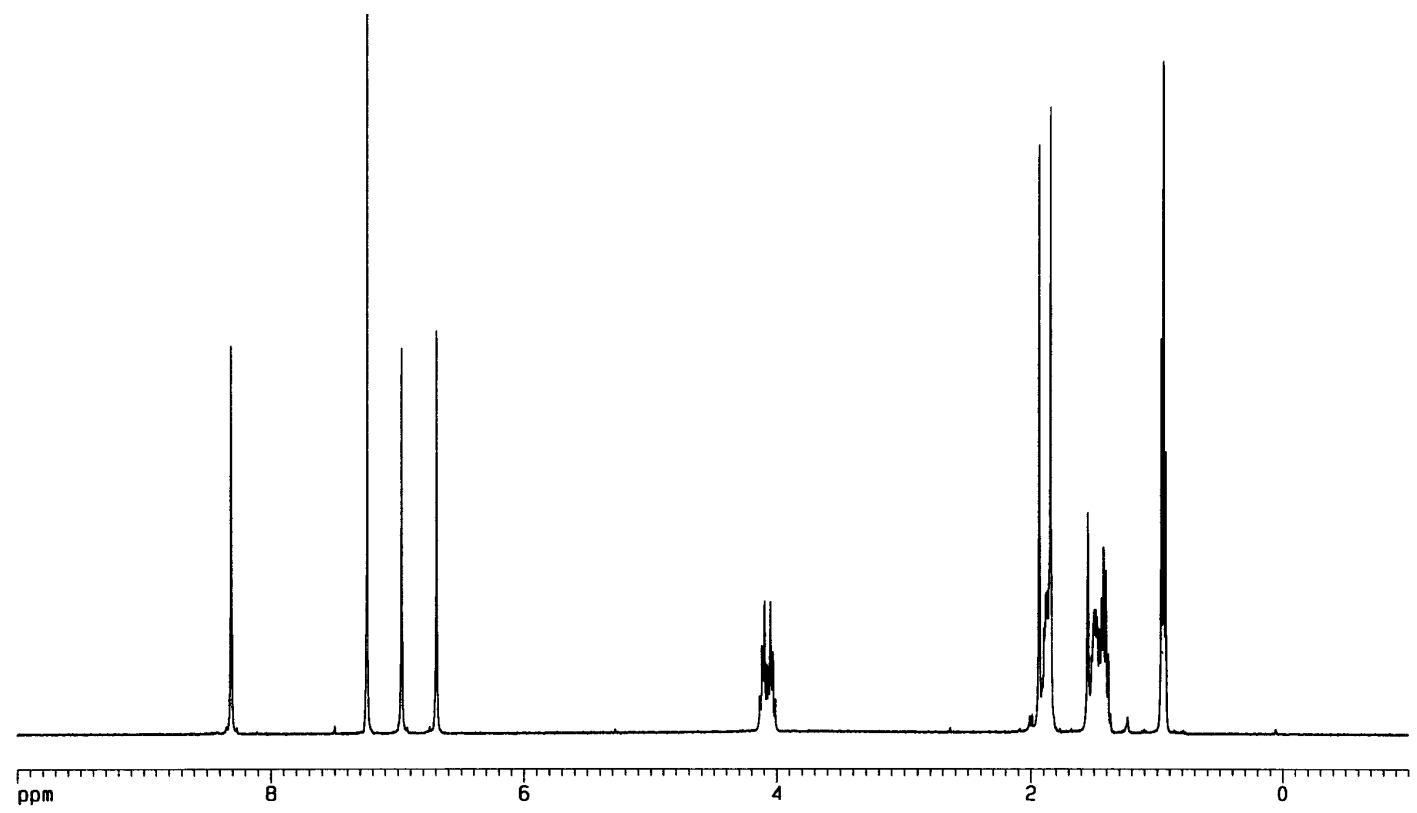

Figure S5. ${ }^{1} \mathrm{H}$ NMR spectrum $\left(400 \mathrm{MHz}, \mathrm{CDCl}_{3}\right)$ of $3 \mathbf{b}\left(\mathrm{R}=\mathrm{O}^{\mathrm{n}} \mathrm{C}_{5} \mathrm{H}_{11}\right)$.

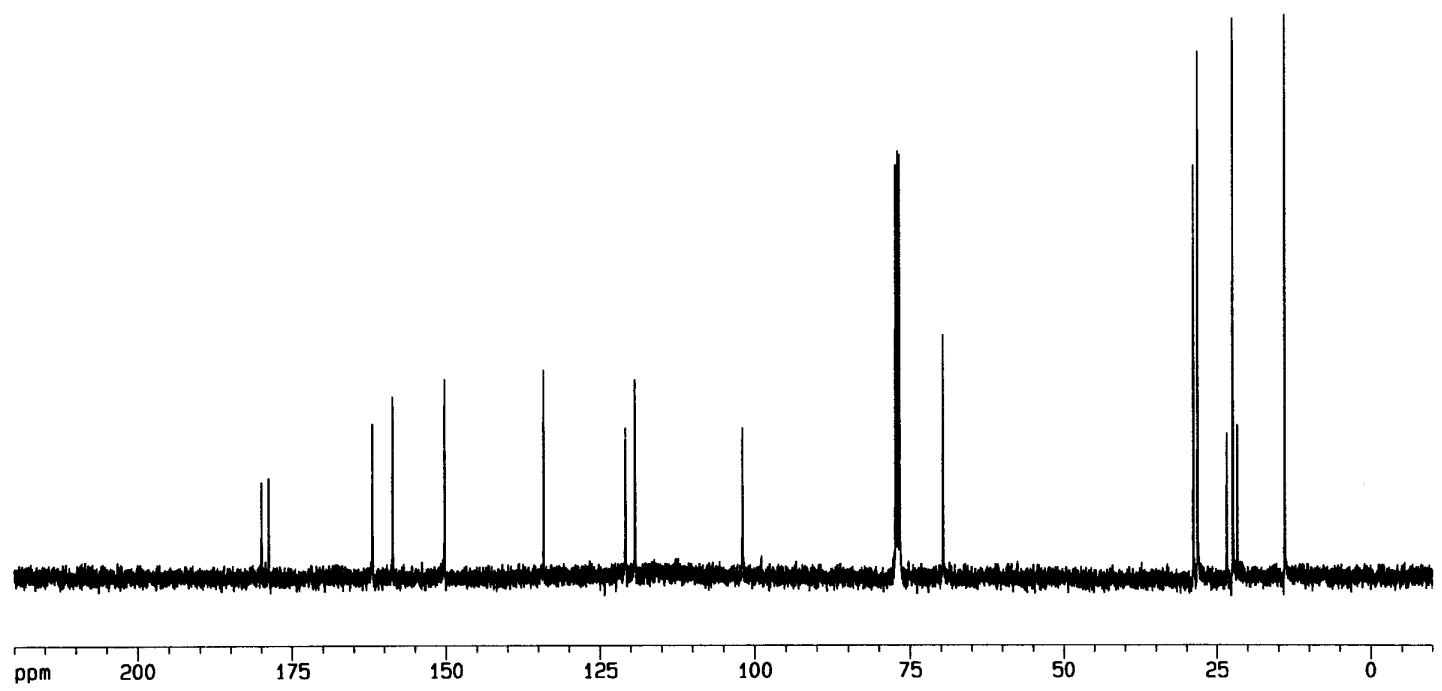

Figure S6. ${ }^{13} \mathrm{C}$ NMR spectrum $\left(100.6 \mathrm{MHz}, \mathrm{CDCl}_{3}\right)$ of $3 \mathbf{b}\left(\mathrm{R}=\mathrm{O}^{\mathrm{n}} \mathrm{C}_{5} \mathrm{H}_{11}\right)$. 


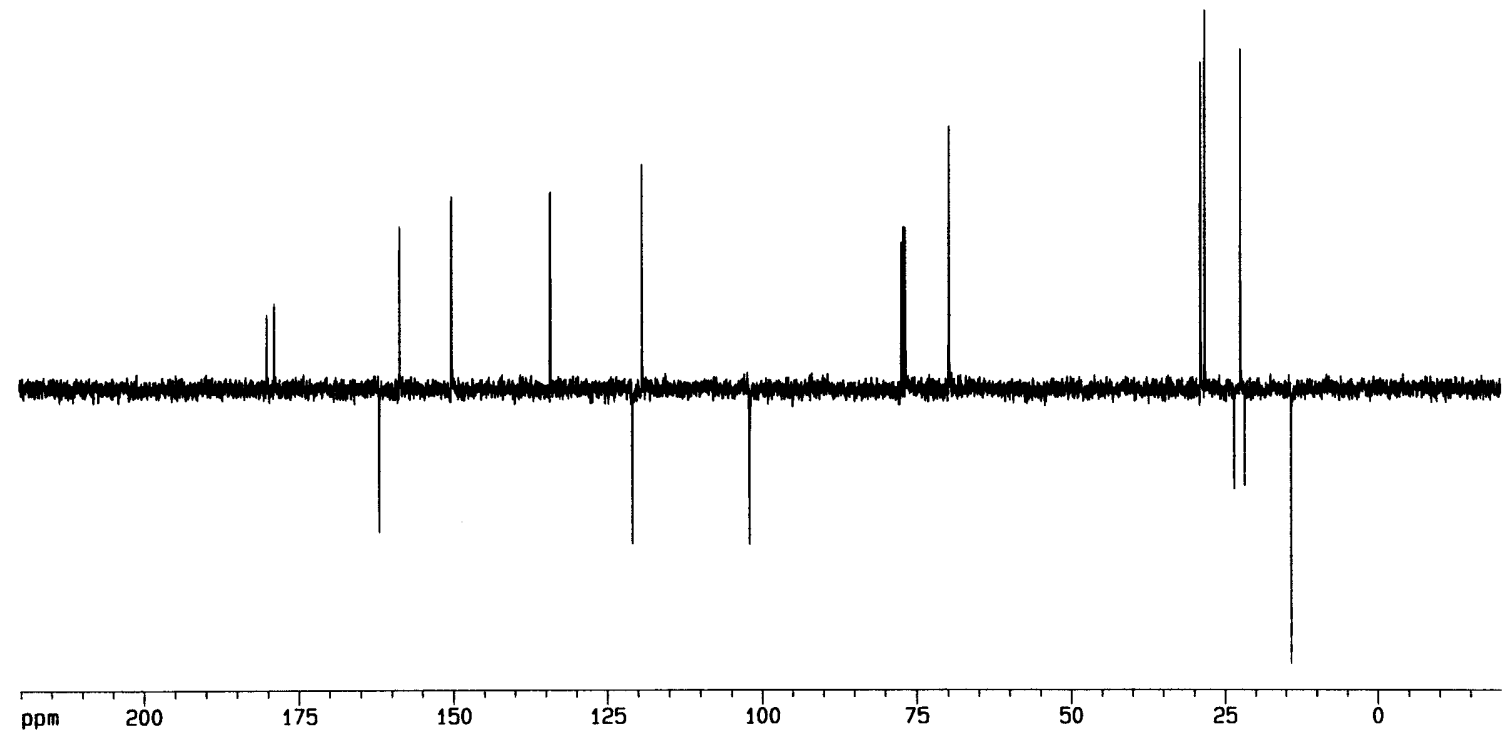

Figure S7. ${ }^{13} \mathrm{C}$ APT NMR spectrum $\left(100.6 \mathrm{MHz}, \mathrm{CDCl}_{3}\right)$ of $\mathbf{3 b}\left(\mathrm{R}=\mathrm{O}^{\mathrm{n}} \mathrm{C}_{5} \mathrm{H}_{11}\right)$.

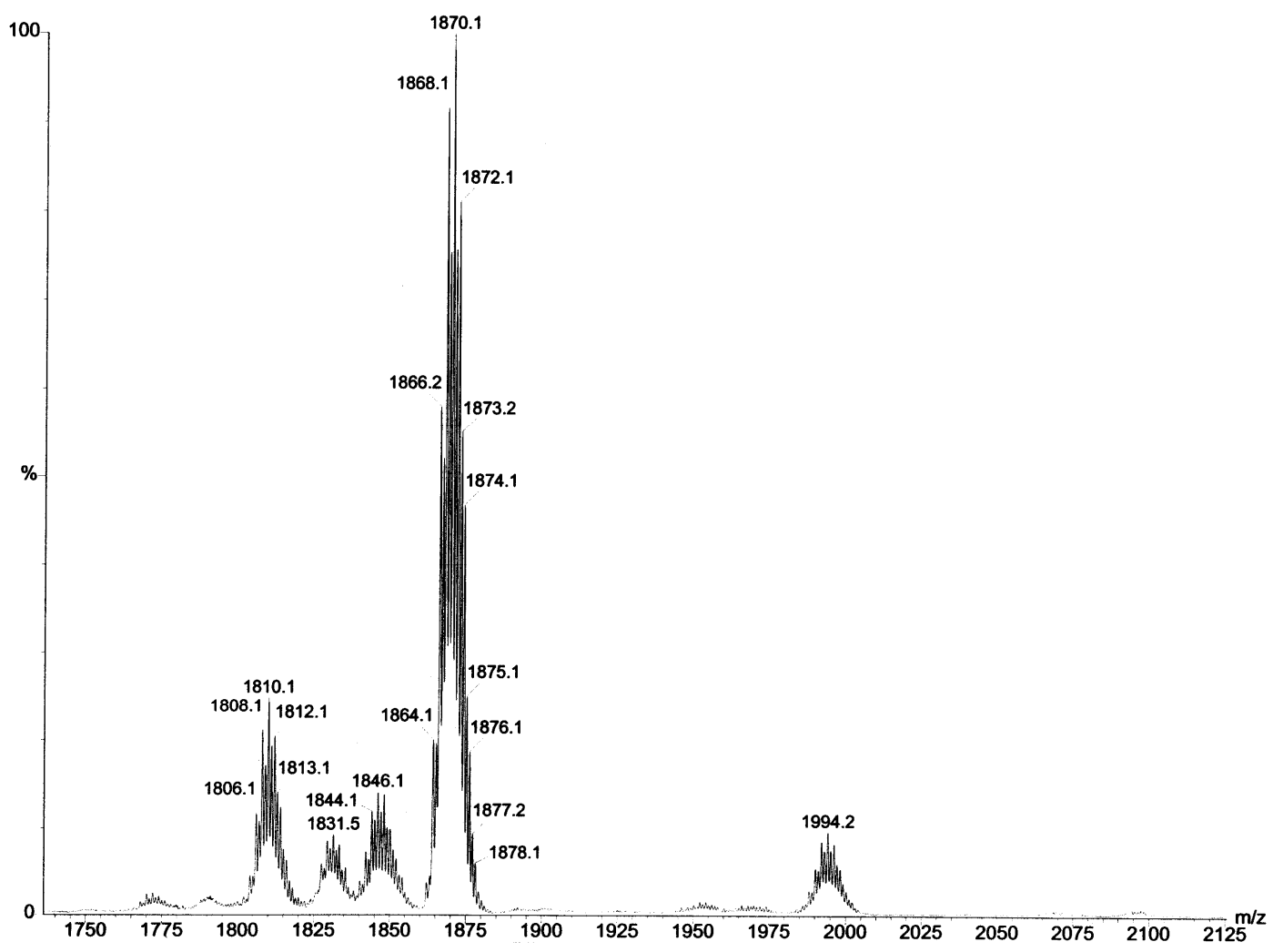

Figure S8. ESI mass spectrum of $3 \mathbf{b}\left(\mathrm{R}=\mathrm{O}^{\mathrm{n}} \mathrm{C}_{5} \mathrm{H}_{11}\right)$. 


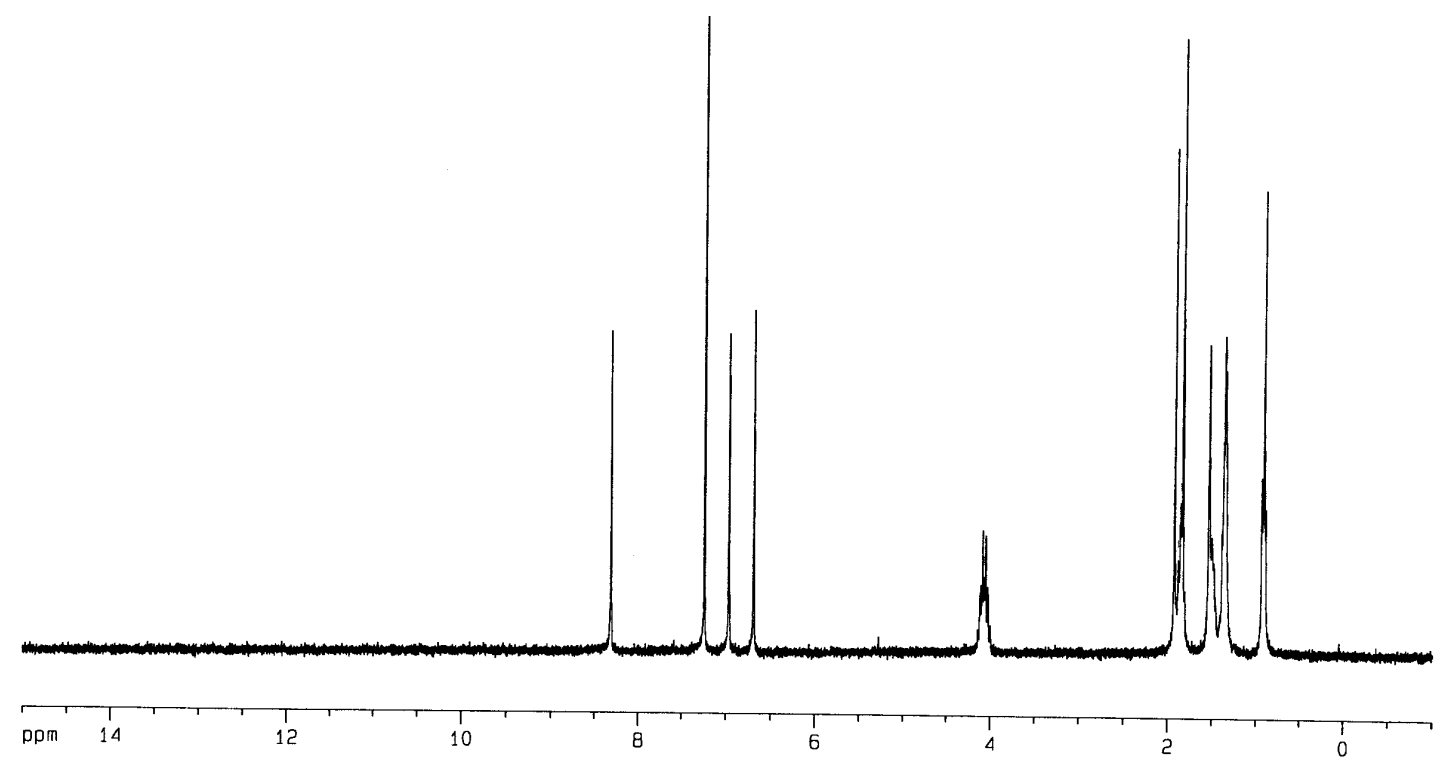

Figure S9. ${ }^{1} \mathrm{H}$ NMR spectrum $\left(300 \mathrm{MHz}, \mathrm{CDCl}_{3}\right)$ of $3 \mathbf{c}\left(\mathrm{R}=\mathrm{O}^{\mathrm{n}} \mathrm{C}_{6} \mathrm{H}_{13}\right)$.

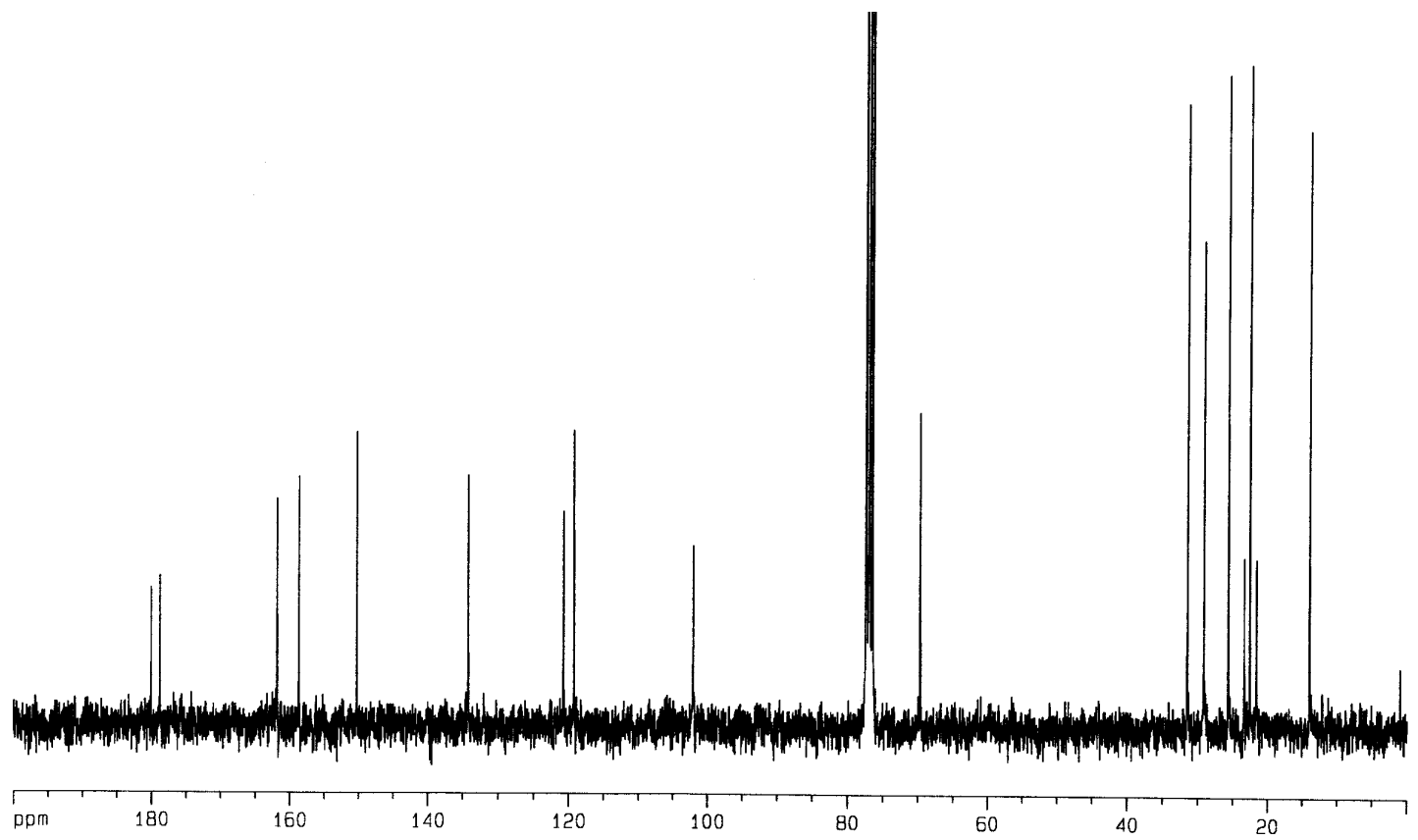

Figure S10. ${ }^{13} \mathrm{C}$ NMR spectrum $\left(75.5 \mathrm{MHz}, \mathrm{CDCl}_{3}\right)$ of $3 \mathrm{c}\left(\mathrm{R}=\mathrm{O}^{\mathrm{n}} \mathrm{C}_{6} \mathrm{H}_{13}\right)$. 


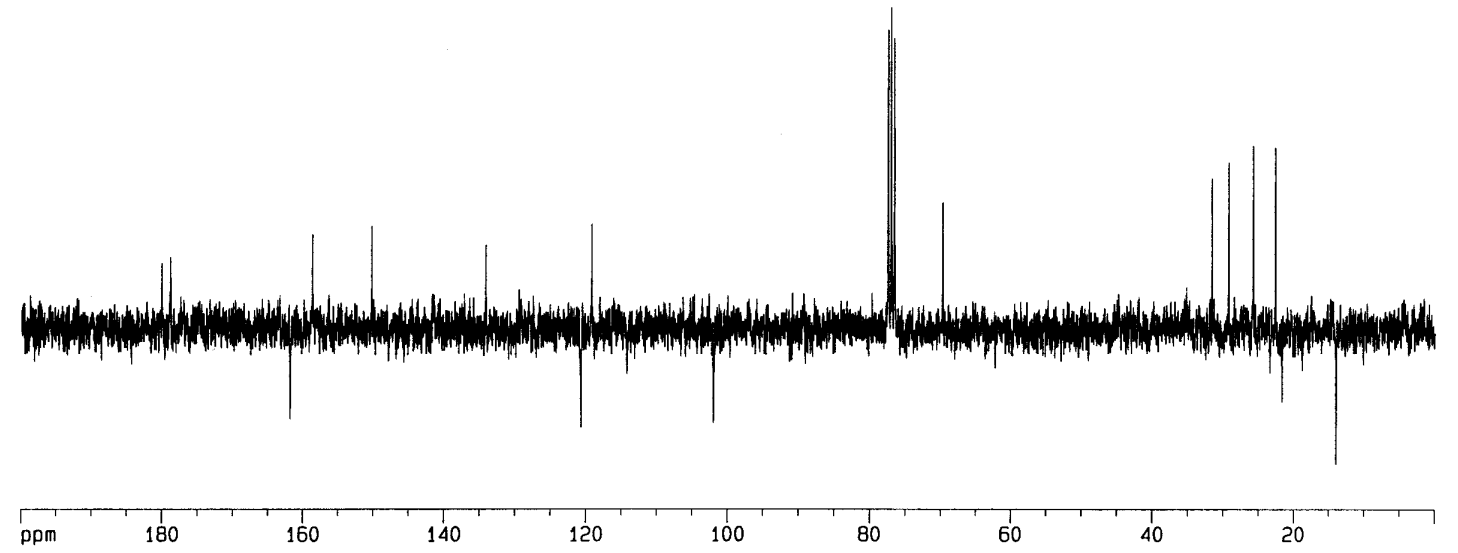

Figure S11. ${ }^{13} \mathrm{C}$ APT NMR spectrum $\left(75.5 \mathrm{MHz}, \mathrm{CDCl}_{3}\right)$ of $3 \mathrm{c}\left(\mathrm{R}=\mathrm{O}^{\mathrm{n}} \mathrm{C}_{6} \mathrm{H}_{13}\right)$.

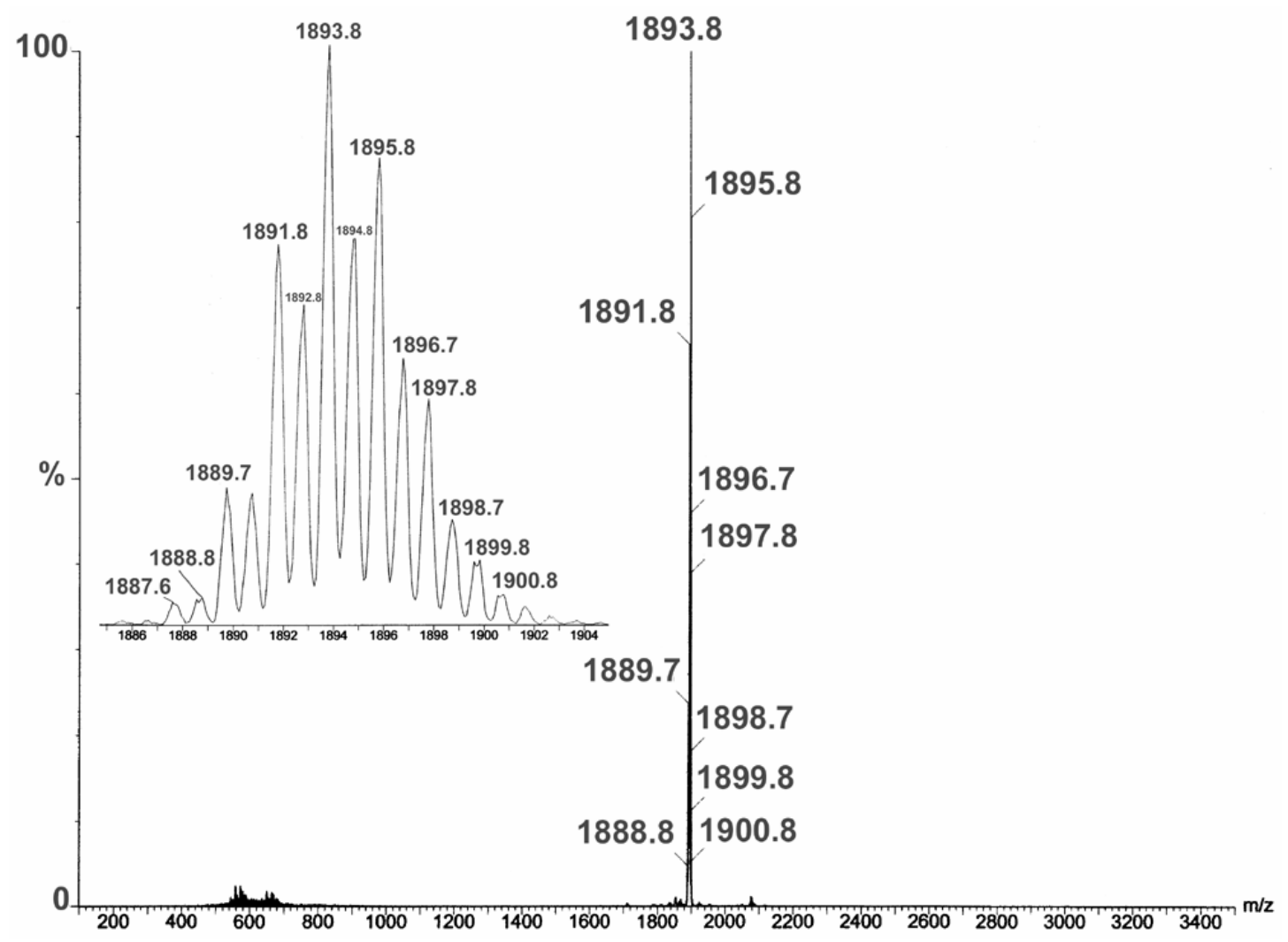

Figure S12. ESI-MS spectrum of 3c (inset: isotope distribution). 